\title{
Some Memories and Dreams from the Childhood of Particle Accelerators
}

\author{
R. Wideröe, Nussbaumen
}

My first interest in atomic structure might have started nearly 70 years ago; I had read somewhere about the planetary system with the positive nucleus in the centre, encircled by negative electrons. The great problem of that time was of course to "split the atom" and I imagined that very strong magnetic fields could do the trick by squeezing the electron orbits so close to the nucleus that reactions might occur: something like a "super-Zeeman" effect. But then in 1919 came the news that Rutherford had split nitrogen atoms with highly energetic $\alpha$-particles from radium. This news made a great impression on me. For the first time the barrier of the nucleus had been broken and the old dream of the alchemists had come true.

However, I saw clearly that the limited flux and energy of $\alpha$-particles made them quite unsuitable in a practical way for splitting the atom. Only electrically accelerated particles with a greatly extended high voltage technology could bring the real solution. With these thoughts in mind I went to Karlsruhe to study electrical engineering. In the autumn of 1922 , I had the idea that electrons in a vacuum vessel could be accelerated in an electrical vortex field, just like the electrons in a coil of a normal

Fig. 2 - Theodor Wideröe (right), seen here with one of his betatrons in 1950, was born in Oslo in 1902. His research took him to Karlsruhe, Aachen, Berlin, and again Oslo before he settled at Brown-Boveri in Baden (Switzerland).

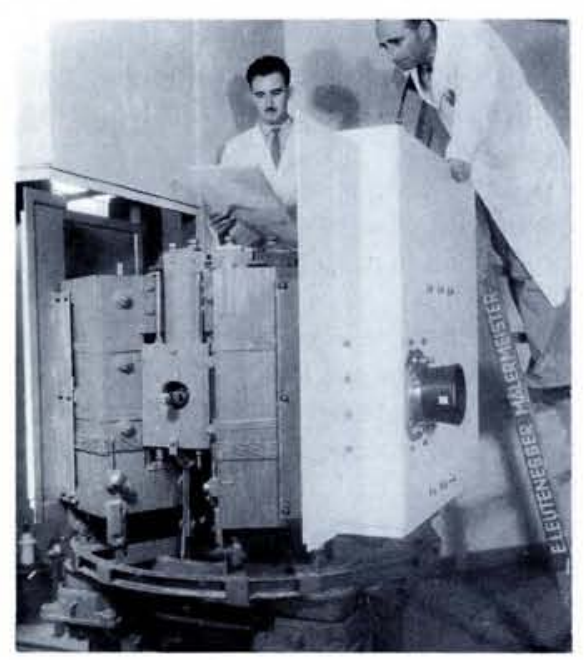

transformer, and thus reach very high voltages. The voltage gained per turn might be small, i.e. only $30 \mathrm{~V}$, but the velocity of the electrons would increase rapidly and soon come close to the velocity of light so that the electrons during the acceleration period would make many orbits and thus reach high energies of many million volts.

Five years later when I had finished my diploma studies I went to Professor Gaede in Karlsruhe with my idea. Gaede, the professor of physics, had invented the diffusion pump for creating high vacua of better than $10^{-6}$ Torr; he was known worldwide as a specialist in high vacuum technology. He gave me a very cold shower. He said: "On the long way the electrons will have to travel before they reach high energy they will all be absorbed by the remaining gas molecules. Your idea will not work, forget it." This was a terrible blow; but "Rocambole was not dead!" In Lenard's paper "Quantitatives über Kathodenstrahlen aller Geschwindigkeiten" (Heidelberger Akademie 1918) I found measured values for absorption cross-sections in air for electron energies from 10 to nearly $10^{6} \mathrm{eV}$ and they showed me that with $10^{-6}$ Torr the absorption would be less than $10 \%$ for an acceleration from 10 to $10^{7} \mathrm{~V}$. The scattering of the electrons by the gas molecules would be of some importance and this might limit the injection energy to not less than about 400 eV. So everything seemed to be fine. It was, however, quite clear that further work in Karlsruhe was impossible.

I next wrote to Professor Rogowski in Aachen. He was then developing very fast cathode ray oscillographs for studies of high voltage surges and he and his assistants had experience with high vacuum. I met Rogowski on a train, travelled with him from Karlsruhe to Mannheim and he agreed with my plans. In Aachen I then built a small "Ray-transformer", my name for the betatron, for 6 MeV. The experiments with this model were, however, not sucessful. I could make the electrons circulate only $1 \frac{1 / 2}{2}$ times in the toroidal vacuum tube. Many stray electrons hit the glass wall and built up surface charges, thus destroying the stability of the orbit. Covering the inner glass walls with a conductive layer

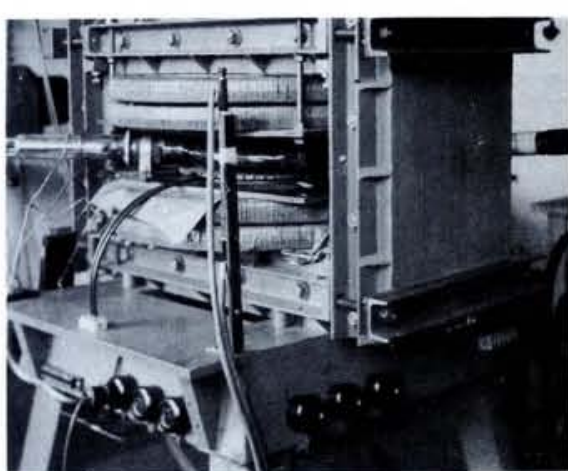

Fig. $1-$ A $15 \mathrm{MeV}$ betatron at the C.H.F. Müller Laboratory in 1944.

of graphite would have solved this minor problem but I had somehow lost my confidence.

Rogowski said: "With a machine that doesn't work you cannot complete a doctorate" and so I built the first linac. The idea I got from a paper by Ising, in which he suggested accelerating particles on the rising front of a travelling high voltage wave. This is not a very easy method; I think it was realized only a few years ago, but I dreamt up the HF excited drift tube and using this "particle lift" my model worked very well. Potassium and sodium ions were accelerated to nearly $60 \mathrm{keV}$ with an accelerating voltage of about $25 \mathrm{kV}$.

However, it was a pity that I gave up the work on my ray-transformer in Aachen. An investigation of my model 25 years later showed that the guiding field of my magnetic structure really was right and the calculations in my notebook from that time show the same results and criteria as Kerst and Serber published 15 years later. My mistake at that time was that I regarded the calculated stabilizing forces as too small and not of any importance - probably the prejudice of an electrical engineer. I was looking for stronger forces, but such forces I only found in 1943, with the "lens way".

I also well remember a day when we, the assistants of the institute, were discussing my work. The late Professor Flegler (he died a year ago) asked a question: "Could not ions be made to circulate, for instance on spiraling orbits, in a magnetic field and be accelerated by HF-excited drift tubes?" My answer was that this was possible but that it would be difficult to stabilize the orbits and that the ions probably would be lost in collisions with the walls. Now, in reality Professor Flegler had invented the cyclotron, but my doubts and prejudices from my experiments with the ray-transformer killed his idea. Therefore: be aware of prejudices! When Lawrence saw my paper he was in the lucky situation that 


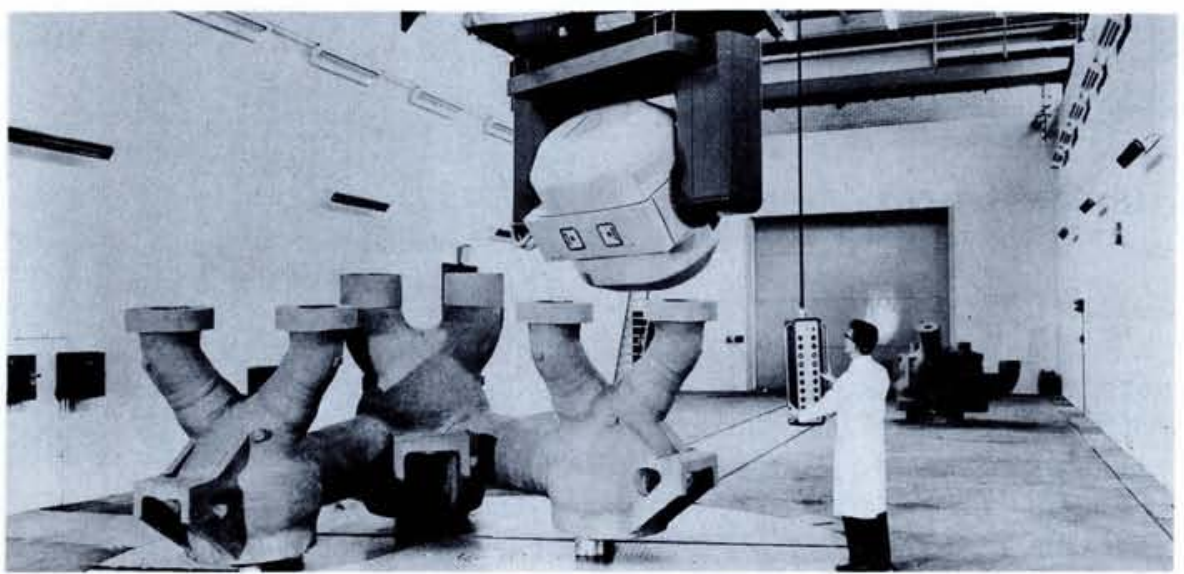

Fig. 3 - Materials testing with a $35 \mathrm{MeV}$ betatron (Photo Brown-Boveri, 1963).

he could not read German and he got his information only from the figures. Therefore he did not learn anything about my stability problems and when he and Livingston built their machine he again was lucky: it worked.

I will now make a long leap to the year 1943. In this and the next year I lived partly in Hamburg and developed at the C.H.F. Müller X-ray factory a small 15 $\mathrm{MeV}$ betatron. The machine, working with 50 cycles from the mains, had a magnetic structure for the guiding field that saturated at the end of acceleration and brought the accelerated electrons to hit a platinum target and produce $\mathrm{X}$-rays. At the end of our work, in January 1945 , the X-ray intensity was $1.2 \mathrm{R} / \mathrm{min}$ at $1 \mathrm{~m}$ from the target. It was the first working betatron outside the USA. The development under war conditions was not easy and it was not possible to try out more advanced ideas. At the end of the war, the betatron was brought to Woolwich Arsenal (near London), reassembled there by my assistant Professor Kollath and used for nondestructive testing of steel plates. From here, the machine later disappeared.

But even if our investigations were severely restricted, our dreams had no limits. While we sat down in the cellar and waited for the bombers to disappear we tried to find solutions to many of the accelerator problems. In 1943 I found a simple way to inject electrons and bring them to a stable orbit (using a superimposed rapidly decaying magnetic field), Electrostatic and magnetic lenses producing strong focussing forces were patented. In addition, a novel, very important method to create nuclear reactions with storage rings and colliding particles (the "nuclear mill") was invented. Shortly after the end of the war, in summer 1945, I found the principle of the synchrotron, i.e. the combination of particles travelling on a circular, constant, orbit accelerated with drift tubes and HF-electric fields. The method, in which the phase focussing of the particles is essential, can be used for the acceleration of electrons and also ions. Here I will only mention a few details which today are used on most synchrotrons: adjusting the acceleration frequency by means of the orbiting particles ("phase lock"), use of $\lambda / 2$ and $\lambda / 4$ resonators, tuning the resonators by ferromagnetic materials and making the accelerating frequency a multiple of the rotational frequency.

The first real development of a betatron for medical and industrial applications started in August 1946 at the Brown Boveri works in Baden (Switzerland). My first working place was far from splendid. In the assembly and testing hall for large electrical generators there were inspection tunnels below the machines, and in one of these tunnels the first betatron was set up. The terrible noise, the draught and the smell when the machine over our heads was started up was quite unbelievable and today I still cannot quite understand how we managed to make the betatron work. However, after solving a lot of problems it really did work and after a while the radiation level became quite dangerous for us; our leucocyte counts dropped and even in the assembly hall radiation intensities increased.

This was of course decisive and therefore we got a usable and safe laboratory for our further work. The development work for a betatron is $95 \%$ perspiration and $5 \%$ inspiration but sometimes you need the inspiration very much. We made the vacuum vessel of $\mathrm{C} 9$ borosilicate glass moulded and blown in a graphite form, coated with "Aquadag" on the inside and sealed off when high vacuum had been obtained. Such tubes performed very well. The magnetic structure must be easy to take apart and re-assemble (when the glass tube has to be changed) and it must be very sturdy so that the machine can be turned around without any deformation. Optimal adjustment of the guiding fields is the key to high performance. The magnetic fields tend to have different phases around the orbit, and at injection when the fields are small this will cause different magnitudes of the fields and consequently fairly big disturbances. We adjusted the field phases of the six yokes and wound equalizing coils on the glass tube. We also had a strange experience. Once we had tried out a spiral winding on the toroidal tube but the azimuthal magnetic fields created were not helpful so we removed it. But then the output dropped to nearly zero. The machine obviously liked the open winding and thereafter we always wound a resistance wire around the tube. This was our "black magic". I think that the injection process might produce unequal space charge distributions around the orbit (bunches) and that mirror images in the spiral winding might have a smoothing influence. The injection scheme from 1943 has worked well and increases the output by a factor of 3-4.

Between the poles for the inductive and guiding fields we have two coils for changing the radius of the orbit. By exciting those coils we may expand or compress the orbit and thus terminate the acceleration process. In the coils, a voltage of about $3 \mathrm{kV}$ is induced and by means of thyratrons the coils are switched to load or unload a condenser. We thus get a current pulse which changes the orbit radius. But this circuit is quite tricky. If we trigger the thyratrons at the wrong time, for voltage phases between 90 and 180 degrees, we get overvoltages which build up without limit and may be very destructive. We had a lot of trouble before we understood and cured this bad habit.

When the acceleration was terminated, the electrons could either be brought to hit an internal target and produce X-rays or they could be brought out of the vacuum tube into free air. For this we used a very simple method. I had previously calculated the influence of a local radial disturbing force on the path of the orbiting electrons and I found that the electrons reacted by creating a bulge against the force; (a typical reaction of many people too!). If the force is directed to the inside of the tube and also decreases in the outward direction, the outward bulge in the electron path will increase in a resonant manner with each revolution and will very soon bring the electrons out of the tube. This theory 
worked very well and about $90 \%$ of the electrons could be brought out in a fairly well collimated beam.

The lifetime of the vacuum tube is mainly determined by the cathode of the electron gun, and here we had great problems. Our first barium oxide coated nickel cathodes had a lifetime of about 500 to 1000 hours. After some years and many experiments we found the solution in dispenser cathodes which we developed together with the Philips works in Eindhoven. The cathode is a small cylindrical tube, flattened on the front side, $20 \mathrm{~mm}$ long and $1 \mathrm{~mm}$ in diameter. The tube, made of sintered tungsten powder, is porous and is filled with melted barium aluminate. Inside the tube there is a platinum heater insulated from the tungsten by a tube of alumina. When the cathode is heated to between 1000 and $1100^{\circ} \mathrm{C}$, barium oxide will diffuse to the surface of the cathode and create a layer with high electron emissivity. Pulsed currents exceeding $300 \mathrm{~mA}$ can easily be obtained and the diffusion of barium oxide to the surface just matches the loss from the surface by bombarding ions. Such cathodes have useful lifetimes surpassing 20000 hours and this explains the long times our betatrons, which have two electron guns, can work without a tube change. I think that the betatron at the Queen Elizabeth Hospital in Kowloon (Hong Kong) might hold the record; it has now worked since 1964 without any interruption.

The vacuum is controlled by an electrically heated titanium getter. The energy of the first betatron in 1951 was $31 \mathrm{MeV}$; without increasing the orbit radius of $24 \mathrm{~cm}$ the energy has now been raised to $45 \mathrm{MeV}$. The output in dose intensity of the X-rays is about 400 times greater than for the Hamburg betatron.

In the late 50 s we also developed a $100 \mathrm{MeV}$ electron-synchrotron for the University of Turin. As proposed by H.C. Pollock we used a betatron starting phase which accelerated the electrons to $2 \mathrm{MeV}(\beta=0.98)$ and then an HFresonator which increased the energy to $100 \mathrm{MeV}$. It was interesting but often difficult work and we learned quite a lot of things. The betatron accelerating field was flux-forced and excited by a special resonant circuit at about $500 \mathrm{~Hz}$. The condenser for this circuit was switched and later re-charged by thyratrons. For the HF cavity we used a $\lambda / 2$ resonator with silver painted electrodes on both sides of the glass wall. This arrangement had in our case great advantages compared with $\lambda / 4$ resonators which are

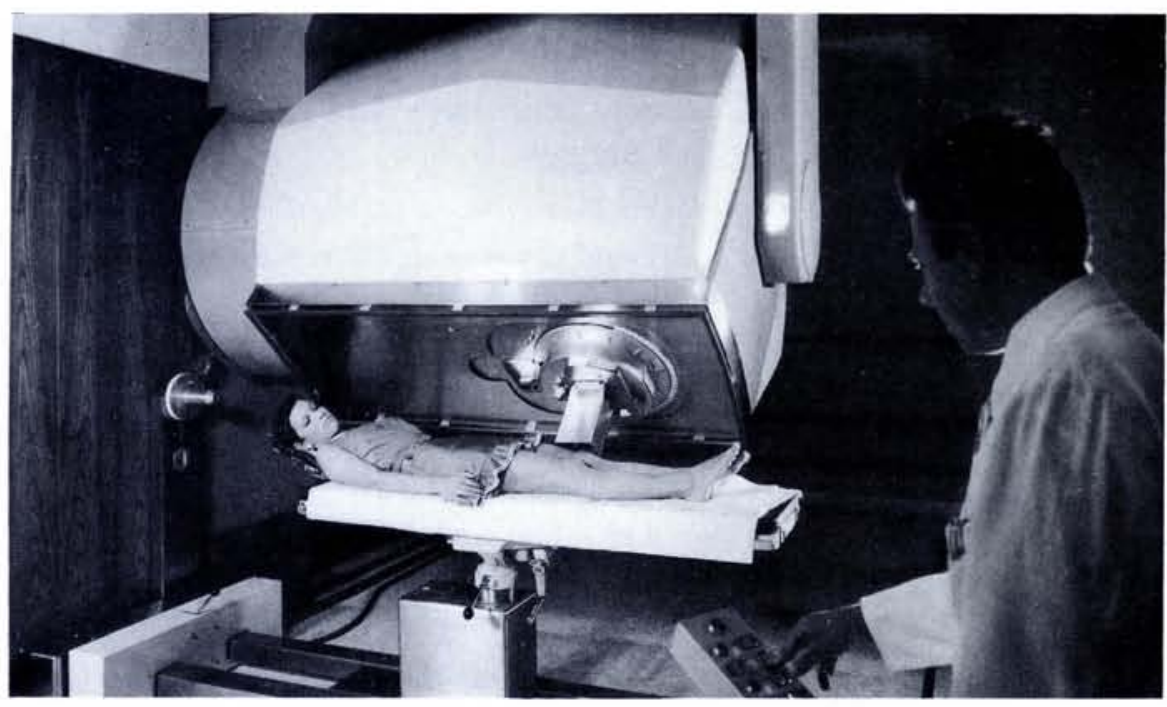

Fig. $4-A$ betatron of $45 \mathrm{MeV}$ in use at a hospital in Zurich (Photo Brown-Boveri, 1970). often used. Most important was that no conductors for HF currents had to be brought through the glass wall. We also had two acceleration gaps each with only $70 \%$ of the voltage needed for a single gap and therefore smaller local losses. The betatron starting phase worked well but when we brought into the machine a tube with an HF resonator the output was zero. After many investigations we painted the inside silver electrode with Aquadag and then the machine worked. I suppose that some of the injected electrons hit the silvered surface of the resonator and created many secondary electrons which then acted like a cork of space charge and closed the tube to the circulating electrons. The carbon surface creates much less secondary electrons and we have the same conditions all around the tube.

Our work with the synchrotron made us feel that it sometimes is much more difficult to build a small machine (our orbital radius was only $29 \mathrm{~cm}$ ) than a bigger one. But unfortunately the bigger machine is much more expensive.

With the great machines at CERN and in Brookhaven being built and working the particle accelerator had grown up and come of age. Childhood has ended.

\section{Fundamental Processes in Atomic Collision Physics}

A NATO Advanced Study Institute on "Fundamental Processes in Atomic Collision Physics" will be held from 10-21 September 1984 at Santa Flavia, Palermo in Sicily, under the sponsorship of the Scientific Affairs Division of the North Atlantic Treaty Organization.

The objective of the ASI is to bring together scientists (theoretical and experimental physicists as well as quantum chemists) to review fundamental processes in atomic collision physics from low to high energies. Besides theoreticians who will report on modern theories, we shall have a few outstanding experimentalists as Main Lecturers to review the different experimental aspects. In addition to the main lectures, a Symposium on New Trends in Atomic Collisions (honorary chairman, U. Fano, University of Chicago) will be held during which scientists will report on the latest developments in selected fields of research.
Director:
H. Kleinpoppen (Stirling, Scotland)
Co-directors:
J.S. Briggs (Freiburg, Fed. Rep. of Germany)
H.O. Lutz (Bielefeld, Fed. Rep. of Germany)

The cost of double room accommodation and full board is US $\$ 490$.- (half board US $\$ 440$.- single room supplement US $\$ 130 .-$ ) for the full period.

Further information and application forms can be obtained from Professor H. Kleinpoppen,

Atomic Physics Laboratory, University of Stirling, Stirling FK9 4LA Scotland

Deadline for applications: 15 May 1984 